\title{
On Nano Generalized $\beta$ Continuous Functions and Nano Generalized $\beta$ Irresolute Functions in Nano Topological Spaces
}

\author{
S.B.Shalini ${ }^{1}$, K.Indirani ${ }^{2}$ \\ ${ }^{I}$ Research Scholar, Department of Mathematics, Nirmala College for Women, Cbe, India. \\ ${ }^{2}$ Associate Professor, Head of the Department of Mathematics, Nirmala College for Women, Coimbatore, \\ Tamil Nadu, India.
}

\begin{abstract}
The aim of this paper is to introduce Nano generalized $\beta$ continuous function in Nano topological spaces and also we introduce and study the relation between Nano generalized $\beta$ irresolute function and Nano generalized $\beta$ continuous function.
\end{abstract}

Keywords: Nano $\beta$ continuous, Nano generalized $\beta$ continuous and Nano generalized $\beta$ irresolute functions.

\section{Introduction}

In general, continuous function is one of the core concepts in topology. Abd El Monsef et al. [1] introduced the notion of $\beta$-open sets and $\beta$-continuous mappings in topology and further the generalized continuous functions was introduced and studied by Balachandran et al.,[2]. The concept of Nano topology was introduced by Lellis Thivagar [5] which was defined in terms of approximations and boundary regions of a subset of a universe using an equivalence relation on it and he also defined Nano continuous functions, Nano open maps, Nano closed maps and Nano homeomorphisms and their representations in terms of Nano interior and Nano closure. In this paper we introduce Nano generalized $\beta$ continuous function and Nano generalized $\beta$ irresolute function and study some of its relation between them.

\section{Preliminaries}

Definition: 2.1 [5] Let $U$ be the universe, $R$ be an equivalence relation on $U$ and $\tau_{R}(X)=\left\{U, \phi, L_{R}(X), U_{R}(X), B_{R}(X)\right\}$ where $X \subseteq U$. Then $\tau_{R}(X)$ satisfies the following axioms:

i) $U$ and $\phi \in \tau_{R}(X)$.

ii) The union of the elements of any sub collection of $\tau_{R}(X)$ is in $\tau_{R}(X)$.

iii) The intersection of the elements of any finite sub collection of $\tau_{R}(X)$ is in $\tau_{R}(X)$.

Then $\tau_{R}(X)$ is a topology on $U$ called the Nano topology on $U$ with respect to $X,\left(U, \tau_{R}(X)\right)$ is called the Nano topological space.

Definition: 2.2 [5] If $\left(U, \tau_{R}(X)\right)$ is a Nano topological space with respect $X$ where $X \subseteq U$ and if $A \subseteq U$ , then

- The Nano interior of a set $A$ is defined as the union of all Nano open subsets contained in $A$ and is denoted by $N$ int $(A) . N \operatorname{int}(A)$ is the largest Nano open subset of $A$.

- The Nano closure of a set $A$ is defined as the intersection of all Nano closed sets containing $A$ and is denoted by $\operatorname{Ncl}(A) . N \operatorname{cl}(A)$ is the smallest Nano closed set containing $A$.

Definition: 2.3 [2] A function $f:(X, \tau) \rightarrow(Y, \sigma)$ is called

(i) continuous if $f^{-1}(V)$ is open in $(X, \tau)$ for every open set $V$ in $(Y, \sigma)$.

(ii) Semi continuous if $f^{-1}(V)$ is semi open in $(X, \tau)$ for every open set $V$ in $(Y, \sigma)$.

(iii)Pre continuous if $f^{-1}(V)$ is pre open in $(X, \tau)$ for every open set $V$ in $(Y, \sigma)$.

(iv) $\alpha$ continuous if $f^{-1}(V)$ is $\alpha$ open in $(X, \tau)$ for every open set $V$ in $(Y, \sigma)$. 
(v) Regular continuous if $f^{-1}(V)$ is regular open in $(X, \tau)$ for every open set $V$ in $(Y, \sigma)$.

(vi) $\quad \beta$ (Semi pre) continuous if $f^{-1}(V)$ is $\beta$ (semi pre) open in $(X, \tau)$ for every open set $V$ in $(Y, \sigma)$.

(vii) $\mathrm{g}$ continuous if $f^{-1}(V)$ is g open in $(X, \tau)$ for every open set $V$ in $(Y, \sigma)$.

Definition: 2.4 [3] A subset $A$ of $\left(U, \tau_{R}(X)\right)$ is called Nano generalized closed set (briefly Ng closed) if $\operatorname{Ncl}(A) \subseteq V$ whenever $A \subseteq V$ and $V$ is Nano open in $\left(U, \tau_{R}(X)\right)$.

Definition: 2.5 [7] A subset $A$ of Nano topological space $\left(U, \tau_{R}(X)\right)$ is called Nano generalized $\beta$ closed set (briefly $\mathrm{Ng} \beta$ closed) if $N \beta c l(A) \subseteq V$ whenever $A \subseteq V$ and $V$ is Nano open in $\left(U, \tau_{R}(X)\right)$.

Definition: 2.6 [7] A subset $A$ of a Nano topological space $\left(U, \tau_{R}(X)\right)$ is called Nano generalized $\beta$ open (briefly $\mathrm{Ng} \beta$ open), if its compliment $A^{C}$ is Nano g $\beta$ closed.

The collection of all Nano g $\beta$ open subsets of is denoted by $N g \beta O(U, X)$.

Definition: 2.7 [6] Let $\left(U, \tau_{R}(X)\right)$ and $\left(V, \sigma_{R^{\prime}}(Y)\right)$ be Nano topological spaces. Then a mapping $f:\left(U, \tau_{R}(X)\right) \rightarrow\left(V, \sigma_{R^{\prime}}(Y)\right)$ is called Nano continuous on $U$ if the inverse image of every Nano open set in $V$ is Nano open in $U$.

Definition: 2.8 Let $\left(U, \tau_{R}(X)\right)$ and $\left(V, \sigma_{R^{\prime}}(Y)\right)$ be Nano topological spaces. Then a mapping $f:\left(U, \tau_{R}(X)\right) \rightarrow\left(V, \sigma_{R^{\prime}}(Y)\right)$ is called Nano $\beta$ continuous on $U$ if the inverse image of every Nano open set in $V$ is Nano $\beta$ open in $U$.

\section{Nano Generalized $\beta$ Continuous Functions}

Throughout this paper, $U$ and $V$ are non-empty, finite universe, $X \subseteq U$ and $Y \subseteq V, U / R$ and $V / R^{\prime}$ denote the families of equivalence classes by equivalence relations $R$ and $R^{\prime}$ respectively on $U$ and $V,\left(U, \tau_{R}(X)\right)$ and $\left(V, \sigma_{R^{\prime}}(Y)\right)$ are the Nano topological spaces with respect to $X$ and $Y$ respectively.

Definition: 3.1 Let $\left(U, \tau_{R}(X)\right)$ and $\left(V, \sigma_{R^{\prime}}(Y)\right)$ be Nano topological spaces and a mapping $f:\left(U, \tau_{R}(X)\right) \rightarrow\left(V, \sigma_{R^{\prime}}(Y)\right)$ is called Nano generalized $\beta$ continuous (briefly $N g \beta$ continuous) on $U$ if the inverse image of every Nano open set in $V$ is Nano g $\beta$ open in $U$.

Example: $\quad$ 3.2 Let $U=\{a, b, c\} \quad$ with $U / R=\{\{a, b\},\{c\}\} \quad$ and $\quad X=\{a, b\} . \quad$ Then $\tau_{R}(X)=\{U, \phi,\{a, b\},\{c\}\}$. Let $V=\{x, y, z\}$ with $V / R^{\prime}=\{\{x\},\{y, z\}\}$ and $Y=\{x, z\}$. Then $\sigma_{R^{\prime}}(Y)=\{V, \phi,\{x\},\{y, z\}\}$. Define $f: U \rightarrow V \quad$ and $f \quad$ as $f(a)=x, f(b)=z, f(c)=y$. Then $f^{-1}(\{x\})=\{a\}, f^{-1}(\{y, z\})=\{b, c\}$. Thus $\{a\}$ and $\{b, c\}$ are $N g \beta$ open sets in $U$. That is the inverse image of every Nano open set in $V$ is $N g \beta$ open set in $U$. Therefore $f$ is $N g \beta$ continuous.

Theorem: 3.3 Every Nano $\beta$ continuous maps Nano $g$ continuous.

Proof: Let $f:\left(U, \tau_{R}(X)\right) \rightarrow\left(V, \sigma_{R^{\prime}}(Y)\right)$ be Nano $\beta$ continuous and $B$ is a Nano closed set in $V$. Then $f^{-1}(B)$ is Nano $\beta$ closed in $U$. Since every Nano $\beta$ closed is Nano g $\beta$ closed, $f^{-1}(B)$ is Nano g $\beta$ closed in $U$. Therefore $f$ is Nano $g$ continuous.

Remark: 3.4 The converse of the above theorem need not be true which can be seen from the following example. 
Example: 3.5 Let $U=\{a, b, c, d\} \quad$ with $U / R=\{\{a\},\{b\},\{c\},\{d\}\}$ and $X=\{a, d\}$. Then $\tau_{R}(X)=\{U, \phi,\{a, d\}\}$. Let $V=\{x, y, z, w\}$ with $V / R^{\prime}=\{\{x, y\},\{z\},\{w\}\}$ and $Y=\{x, z\}$. Then $\sigma_{R^{\prime}}(Y)=\{V, \phi,\{z\},\{x, y, z\},\{x, y\}\}$. Define $f: U \rightarrow V \quad$ and $f \quad$ as $f(a)=x, f(b)=z, f(c)=y, f(d)=w$. Then $f$ is Nano g $\beta$ continuous but $f$ is not Nano $\beta$ continuous since $f^{-1}(\{x, y, w\})=\{a, c, d\}$ is not Nano $\beta$ closed in $U$ whereas $\{x, y, w\}$ is Nano closed in $V$. Thus a Nano g $\beta$ continuous function need not be a Nano $\beta$ continuous function.

The following theorem can also be proved in a similar way.

Theorem: 3.6 Let $\left(U, \tau_{R}(X)\right)$ and $\left(V, \sigma_{R^{\prime}}(Y)\right)$ be Nano topological spaces. Then

(i) Every Nano continuous maps Nano g $\beta$ continuous.

(ii) Every Nano semi continuous maps Nano g $\beta$ continuous.

(iii) Every Nano pre continuous maps Nano g $\beta$ continuous.

(iv) Every Nano regular continuous maps Nano g $\beta$ continuous.

(v) Every Nano $\alpha$ continuous maps Nano g $\beta$ continuous.

(vi) Every Nano b continuous maps Nano g $\beta$ continuous.

(vii) Every Nano g continuous maps Nano g $\beta$ continuous.

(viii) Every Nano g s continuous maps Nano $g$ continuous.

(ix) Every Nano $\alpha \mathrm{g}$ continuous maps Nano g $\beta$ continuous.

(x) Every Nano g $\mathrm{r}$ continuous maps Nano g $\beta$ continuous.

Remark: 3.7 Reverse implications of the above theorem 3.6 need not be true which can be seen from the following example.

Example: 3.8 Let $U=\{a, b, c, d\}$ with $U / R=\{\{a\},\{c\},\{b, d\}\}$ and $X=\{a, b\}$. Then $\tau_{R}(X)=\{U, \phi,\{a\},\{a, b, d\},\{b, d\}\}$.Let $\quad V=\{x, y, z, w\} \quad$ with $\quad V / R^{\prime}=\{\{x, y\},\{z\},\{w\}\} \quad$ and $Y=\{x, z\}$. Then $\sigma_{R^{\prime}}(Y)=\{V, \phi,\{z\},\{x, y, z\},\{x, y\}\}$. Define $f: U \rightarrow V \quad$ and $f \quad$ as $f(a)=z, f(b)=w, f(c)=x, f(d)=y$. Then $f$ is Nano $g \beta$ continuous but $f$ is not Nano continuous, Nano semi continuous, Nano pre continuous, Nano regular continuous, Nano $\alpha$ continuous, Nano b continuous, Nano g continuous, Nano gs continuous, Nano $\alpha \mathrm{g}$ continuous, Nano gr continuous since $f^{-1}(\{z, w\})=\{a, b\}$ is not Nano closed, Nano semi closed, Nano pre closed, Nano regular closed, Nano $\alpha$ closed, Nano b closed, Nano g closed, Nano gs closed, Nano $\alpha$ g closed, Nano gr closed in $U$ whereas $\{z, w\}$ is Nano closed in $V$. Thus a Nano $\mathrm{g} \beta$ continuous function need not be Nano continuous, Nano semi continuous, Nano pre continuous, Nano regular continuous, Nano $\alpha$ continuous, Nano b continuous, Nano g continuous, Nano gs continuous, Nano $\alpha$ g continuous, Nano gr continuous.

We have the following implications for the properties of subsets:

1 Nano $\beta$ continuous, 2 Nano continuous, 3 Nano semi continuous, 4 Nano pre continuous, $\quad 5$ Nano regular continuous, 6 Nano $\alpha$ continuous, 7 Nano b continuous, 8 Nano g continuous, 9 Nano gs continuous, 10 Nano $\alpha$ g continuous, 11 Nano gr continuous. 


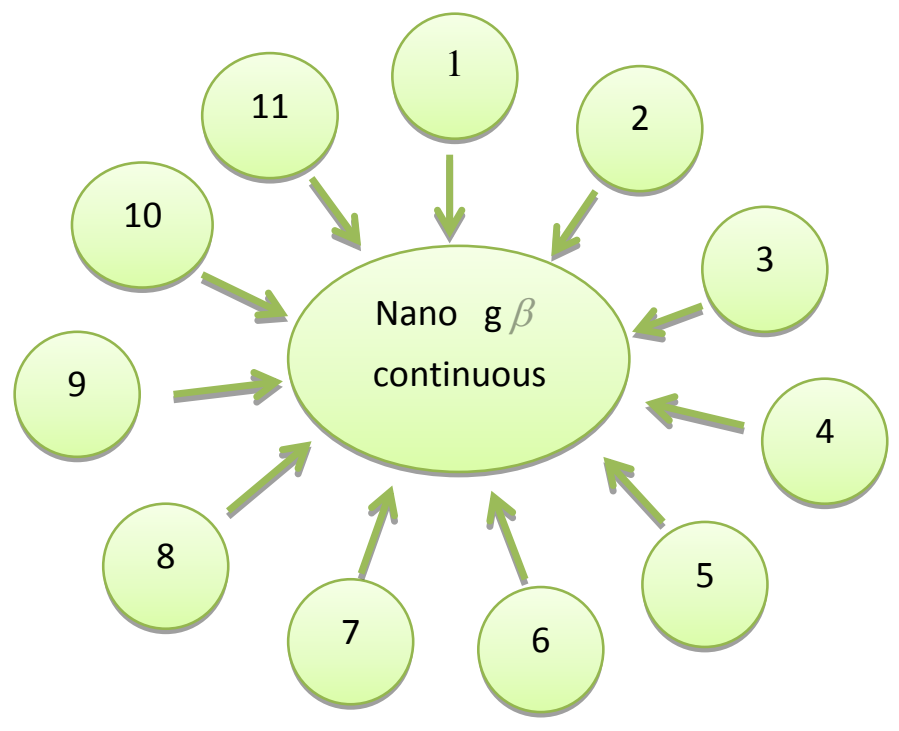

Theorem: 3.9 A function $f:\left(U, \tau_{R}(X)\right) \rightarrow\left(V, \sigma_{R^{\prime}}(Y)\right)$ is $N g \beta$ continuous if and only if the inverse image of every Nano closed set in $V$ is $N g \beta$ closed in $U$.

Proof: Let $f$ be $N g \beta$ continuous and $F$ is Nano closed in $V$. That is $V-F$ is Nano open in $V$. Since $f$ is $N g \beta$ continuous, $f^{-1}(V-F)$ is $N g \beta$ open in $U$. That is $U-f^{-1}(F)$ is $N g \beta$ open in $U$. Therefore $f^{-1}(F)$ is $N g \beta$ closed in $U$. Thus the inverse image of every Nano closed set in $V$ is $N g \beta$ closed in $U$, if $f$ is Nano continuous on $U$.

Conversely, let the inverse image of every Nano closed set be $N g \beta$ closed. Let $G$ is Nano open in $V$. Then $V-G$ is Nano closed in $V$. Then $f^{-1}(V-G)$ is $N g \beta$ closed in $U$. That is $U-f^{-1}(G)$ is $N g \beta$ closed in $U$. Therefore $f^{-1}(G)$ is $N g \beta$ open in $U$. Thus, the inverse image of every Nano open set in $V$ is $N g \beta$ open in $U$. That is, $f$ is $N g \beta$ continuous on $U$.

Theorem: 3.10 A function $f:\left(U, \tau_{R}(X)\right) \rightarrow\left(V, \sigma_{R^{\prime}}(Y)\right)$ is $N g \beta$ continuous if and only if $f(N g \beta c l(A)) \subseteq N c l(f(A))$ for every subset $A$ of $U$.

Proof: Let $f$ be $N g \beta$ continuous and $A \subseteq U$. Then $f(A) \subseteq V$. Since $f$ be $N g \beta$ continuous and $N c l(f(A))$ is Nano closed in $V, f^{-1}(N c l(f(A)))$ is $N g \beta$ closed in $U$.

Since $\quad f(A) \subseteq N c l(f(A)), f^{-1}(f(A)) \subseteq f^{-1}(N c l(f(A))), \quad$ then $\quad A \subseteq f^{-1}(N c l(f(A)))$. $N g \beta c l(A) \subseteq N g \beta c l\left[f^{-1}(N c l(f(A)))\right]=f^{-1}(N c l(f(A)))$. Thus $\quad N g \beta c l(A) \subseteq f^{-1}(N c l(f(A)))$. Therefore $f(N g \beta c l(A)) \subseteq N c l(f(A))$ for every subset $A$ of $U$.

Conversely, let $f(N g \beta c l(A)) \subseteq N c l(f(A))$ for every subset $A$ of $U$. If $F$ is Nano closed in $V$ and since $f^{-1}(F) \subseteq U, f\left(N g \beta c l\left(f^{-1}(F)\right)\right) \subseteq N c l\left(f\left(f^{-1}(F)\right)\right)=N c l(F)=F$.

That is $f\left(N g \beta c l\left(f^{-1}(F)\right)\right) \subseteq F$. Thus $N g \beta c l\left(f^{-1}(F)\right) \subseteq f^{-1}(F)$. But $f^{-1}(F) \subseteq N g \beta c l\left(f^{-1}(F)\right)$.

Hence $N g \beta c l\left(f^{-1}(F)\right)=f^{-1}(F)$. Therefore $f^{-1}(F)$ is $N g \beta$ closed in $U$, for every Nano closed set $F$ in $V$. Thus $f$ is $N g \beta$ continuous.

Remark: 3.11 If $f:\left(U, \tau_{R}(X)\right) \rightarrow\left(V, \sigma_{R^{\prime}}(Y)\right)$ is $N g \beta$ continuous then $f(N g \beta c l(A))$ is not necessarily equal to $\operatorname{Ncl}(f(A))$ where $A \subseteq U$. 
Example: 3.12 Let $U=\{a, b, c, d\}$ with $U / R=\{\{a\},,\{c\},\{b, d\}\}$ and $X=\{a, b\}$. Then $\tau_{R}(X)=\{U, \phi,\{a\},\{b, d\},\{a, b, d\}\}$. Let $\quad V=\{x, y, z, w\} \quad$ with $\quad V / R^{\prime}=\{\{x, y\},\{z\},\{w\}\} \quad$ and $Y=\{x, z\}$. Then $\sigma_{R^{\prime}}(Y)=\{V, \phi,\{z\},\{x, y\},\{x, y, z\}\}$. Define $f: U \rightarrow V \quad$ and $f$ as $f(a)=z, f(b)=x, f(c)=w, f(d)=y . \quad$ Then $\quad f^{-1}(\{z\})=\{a\}, f^{-1}(\{x, y\})=\{b, d\}$, $f^{-1}(\{x, y, z\})=\{a, b, d\}$. Thus $\{a\},\{b, d\}$ and $\{a, b, d\}$ are $N g \beta$ open sets in $U$. That is the inverse image of every Nano open set in $V$ is $N g \beta$ open set in $U$. Therefore $f$ is $N g \beta$ continuous. Let $A=\{b, c\} \subseteq U . \quad$ Then $\quad f(\operatorname{Ng} \beta C l(A))=f(\operatorname{Ng} \beta C l(\{b, c\}))=f(\{b, c\})=\{x, w\} \quad$. But $N C l(f(A))=N C l(\{x, w\})=\{x, y, w\}$. Thus $f(N g \beta C l(A)) \neq N C l(f(A))$, even though $f$ is $N g \beta$ continuous. That is equality does not hold in the previous theorem when $f$ is $N g \beta$ continuous.

Theorem: 3.13 A function $f:\left(U, \tau_{R}(X)\right) \rightarrow\left(V, \sigma_{R^{\prime}}(Y)\right)$ is $N g \beta$ continuous if and only if $N g \beta c l\left(f^{-1}(B)\right) \subseteq f^{-1}(N c l(B))$ for every subset $B$ of $V$.

Proof: If $f$ is $N g \beta$ continuous and $B \subseteq V$. Ncl $(B)$ is Nano closed in $V$ and hence, $f^{-1}(N c l(B))$ is $N g \beta$ closed in $U$. Therefore $N g \beta c l\left(f^{-1}(N c l(B))\right)=f^{-1}(\operatorname{Ncl}(B))$. Since $B \subseteq N c l(B)$, $f^{-1}(B) \subseteq f^{-1}(N c l(B))$. Therefore $N g \beta c l\left(f^{-1}(B)\right) \subseteq N g \beta c l\left(f^{-1}(N c l(B))\right)=f^{-1}(N c l(B))$. That is $N g \beta c l\left(f^{-1}(B)\right) \subseteq f^{-1}(N c l(B))$.

Conversely let $N g \beta c l\left(f^{-1}(B)\right) \subseteq f^{-1}(N c l(B))$ for every subset $B$ of $V$. If $B$ is Nano closed in $V$, then $N c l(B)=B . \quad$ By $\quad$ assumption, $\quad N g \beta c l\left(f^{-1}(B)\right) \subseteq f^{-1}(N c l(B))=f^{-1}(B) \quad$. Thus $N g \beta c l\left(f^{-1}(B)\right) \subseteq f^{-1}(B)$. But $f^{-1}(B) \subseteq N g \beta c l\left(f^{-1}(B)\right)$. Therefore $N g \beta c l\left(f^{-1}(B)\right)=f^{-1}(B)$.

Hence $f^{-1}(B)$ is $N g \beta$ closed in $U$, for every Nano closed set $B$ in $V$. Therefore $f$ is $N g \beta$ continuous on $U$.

Remark: 3.14 If $f:\left(U, \tau_{R}(X)\right) \rightarrow\left(V, \sigma_{R^{\prime}}(Y)\right)$ is $N g \beta$ continuous then $N g \beta c l\left(f^{-1}(B)\right)$ is not necessarily equal to $f^{-1}(N c l(B))$ where $B \subseteq V$.

Example: $\quad 3.15$ Let $U=\{a, b, c, d\}$ with $U / R=\{\{a, b\},\{c\},\{d\}\}$ and $X=\{a, c\}$. Then $\tau_{R}(X)=\{U, \phi,\{c\},\{a, b\},\{a, b, c\}\} . \quad$ Let $V=\{x, y, z, w\} \quad$ with $V / R^{\prime}=\{\{x\},\{y\},\{z\},\{w\}\} \quad$ and $Y=\{x, w\}$. Then $\sigma_{R^{\prime}}(Y)=\{V, \phi,\{x, w\}\}$. Define $f: U \rightarrow V \quad$ and $f \quad$ as $f(a)=y, f(b)=x, f(c)=w, f(d)=z$. Then $\quad f^{-1}(V)=U, f^{-1}(\phi)=\{\phi\}, f^{-1}(\{x, w\})=\{\{b, c\}\}$. Thus $\{b, c\}$ is $N g \beta$ open sets in $U$. That is the inverse image of every Nano open set in $V$ is $N g \beta$ open set in $U$. Therefore $f$ is $N g \beta$ continuous. Let $B=\{x\} \subseteq V$. Then $N g \beta C l\left(f^{-1}(B)\right) \subseteq N g \beta C l\left(f^{-1}(\{x\})\right)=\operatorname{Ng} \beta C l(\{b\})=\{b\}$.

But $f^{-1}(N C l(B))=f^{-1}(N C l(\{x\}))=f^{-1}(V)=U$. Thus $N g \beta C l\left(f^{-1}(B)\right) \neq f^{-1}(N C l(B))$, even though $f$ is $N g \beta$ continuous. That is equality does not hold in the previous theorem when $f$ is $N g \beta$ continuous.

Theorem: 3.16 A function $f:\left(U, \tau_{R}(X)\right) \rightarrow\left(V, \sigma_{R^{\prime}}(Y)\right)$ is $N g \beta$ continuous if and only if $f^{-1}(N$ int $(B)) \subseteq N g \beta$ int $\left(f^{-1}(B)\right)$ for every subset $B$ of $V$.

Proof: If $f$ is $N g \beta$ continuous and $B \subseteq V . N$ int $(B)$ is Nano open in $V$ and hence, $f^{-1}(N$ int $(B))$ is $N g \beta$ open in $U$. Therefore $N g \beta \operatorname{int}\left(f^{-1}(N \operatorname{int}(B))\right)=f^{-1}(N \operatorname{int}(B))$. Also $N \operatorname{int}(B) \subseteq B$,implies that 
$f^{-1}(N$ int $(B)) \subseteq f^{-1}(B) . \quad$ Therefore $\quad N g \beta \operatorname{int}\left(f^{-1}(N \operatorname{int}(B))\right) \subseteq N g \beta \operatorname{int}\left(f^{-1}(B)\right) . \quad$ That is $f^{-1}(N$ int $(B)) \subseteq N g \beta$ int $\left(f^{-1}(B)\right)$.

Conversely let $f^{-1}(N \operatorname{int}(B)) \subseteq N g \beta$ int $\left(f^{-1}(B)\right)$ for every subset $B$ of $V$. If $B$ is Nano open in $V$, then $\quad N \operatorname{int}(B)=B$. By assumption, $\quad f^{-1}(N \operatorname{int}(B)) \subseteq N g \beta \operatorname{int}\left(f^{-1}(B)\right) \quad$. Thus $f^{-1}(B) \subseteq N g \beta$ int $\left(f^{-1}(B)\right)$. But $N g \beta$ int $\left(f^{-1}(B)\right) \subseteq f^{-1}(B)$. Therefore $N g \beta$ int $\left(f^{-1}(B)\right)=f^{-1}(B)$

That is, $f^{-1}(B)$ is $N g \beta$ open in $U$, for every Nano open set $B$ in $V$. Therefore $f$ is $N g \beta$ continuous on $U$.

Remark: 3.17 If $f:\left(U, \tau_{R}(X)\right) \rightarrow\left(V, \sigma_{R^{\prime}}(Y)\right)$ is $N g \beta$ continuous then $N g \beta$ int $\left(f^{-1}(B)\right)$ is not necessarily equal to $f^{-1}(N$ int $(B))$ where $B \subseteq V$.

Example: 3.18 Let $U=\{a, b, c, d\} \quad$ with $U / R=\{\{a\},,\{c\},\{b, d\}\} \quad$ and $\quad X=\{a, b\}$. Then $\tau_{R}(X)=\{U, \phi,\{a\},\{b, d\},\{a, b, d\}\}$.Let $\quad V=\{x, y, z, w\} \quad$ with $\quad V / R^{\prime}=\{\{x, y\},\{z\},\{w\}\} \quad$ and $Y=\{x, z\}$. Then $\sigma_{R^{\prime}}(Y)=\{V, \phi,\{z\},\{x, y\},\{x, y, z\}\}$. Define $f: U \rightarrow V$ and $f \quad$ as $f(a)=z, f(b)=x, f(c)=w, f(d)=y . \quad$ Then $\quad f^{-1}(\{z\})=\{a\}, f^{-1}(\{x, y\})=\{b, d\}$, $f^{-1}(\{x, y, z\})=\{a, b, d\}$. Thus $\{a\},\{b, d\}$ and $\{a, b, d\}$ are $N g \beta$ open sets in $U$. That is the inverse image of every Nano open set in $V$ is $N g \beta$ open set in $U$. Therefore $f$ is $N g \beta$ continuous. Let $B=\{y, z\} \subseteq V$. Then $N g \beta$ int $\left(f^{-1}(B)\right) \subseteq N g \beta$ int $\left(f^{-1}(\{y, z\})\right)=N g \beta$ int $(\{a, d\})=\{a, d\}$. But $f^{-1}(N \operatorname{int}(B))=f^{-1}(N \operatorname{int}(\{y, z\}))=f^{-1}(\{z\})=\{a\}$. Thus $N g \beta$ int $\left(f^{-1}(B)\right) \neq f^{-1}(N$ int $(B))$, even though $f$ is $N g \beta$ continuous. That is equality does not hold in the previous theorem when $f$ is $N g \beta$ continuous.

Theorem: 3.19 If $\left(U, \tau_{R}(X)\right)$ and $\left(V, \sigma_{R^{\prime}}(Y)\right)$ are Nano topological spaces with respect to $X \subseteq U$ and $Y \subseteq V$ respectively, then for any function $f: U \rightarrow V$, the following are equivalent. i) $f$ is $N g \beta$ continuous

ii) the inverse image of every Nano closed set in $V$ is $N g \beta$ closed in $U$

iii) $f(N g \beta c l(A)) \subseteq N c l(f(A))$ for every subset $A$ of $U$

iv) $N g \beta c l\left(f^{-1}(B)\right) \subseteq f^{-1}(N c l(B))$ for every subset $B$ of $V$

v) $f^{-1}(N$ int $(B)) \subseteq N g \beta$ int $\left(f^{-1}(B)\right)$ for every subset $B$ of $V$.

Proof follows from the theorems 3.9, 3.10, 3.13, 3.16.

\section{Relation Between N G $\beta$ Continuous Map And Ng $\beta$ Irresolute Map}

Analogous to irresolute maps in Nano topological spaces we introduce the class of $N g \beta$ irresolute maps which is included in the class of $N g \beta$ continuous maps. In this section we investigate basic properties of $N g \beta$ irresolute maps.

Definition: 4.1 Let $\left(U, \tau_{R}(X)\right)$ and $\left(V, \sigma_{R^{\prime}}(Y)\right)$ be Nano topological spaces and a mapping $f:\left(U, \tau_{R}(X)\right) \rightarrow\left(V, \sigma_{R^{\prime}}(Y)\right)$ is called Nano generalized $\beta$ irresolute (briefly $N g \beta$ irresolute) if the inverse image of every Nanog $\beta$ closed set in $V$ is Nano g $\beta$ closed in $U$.

Example: 4.2 Let $U=\{x, y, z\}$ with $U / R=\{\{x\},\{y, z\}\}$ and $X=\{x, z\}$. Then the Nano topology is defined as $\tau_{R}(X)=\{U, \phi,\{x\},\{y, z\}\}$. Let $V=\{a, b, c\}$ with $V / R^{\prime}=\{\{a, b\},\{c\}\}$ and $Y=\{a, b\}$. Then 
$\sigma_{R^{\prime}}(Y)=\{V, \phi,\{x\},\{y, z\}\}$. Define $f: U \rightarrow V$ and $f(x)=a, f(y)=c, f(z)=b$. Then $f$ is Nano $g$ $\beta$ irresolute since the inverse image of every Nano g $\beta$ closed set in $V$ is Nano g $\beta$ closed in $U$.

Theorem: 4.3 A function $f: U \rightarrow V$ is Nano g $\beta$ irresolute, then $f$ is Nano $g \beta$ continuous.

Proof: Let $f: U \rightarrow V$ is Nano g $\beta$ irresolute, then the inverse image of every Nano $g$ closed set in $V$ is Nano g $\beta$ closed in $U$. Let $F$ be Nano closed in $V$, then $F$ is Nano $g \beta$ closed in $V$ and $f$ is Nano $g$ irresolute. Hence $f^{-1}(F)$ is Nano g $\beta$ closed. Therefore $f$ is Nano $g$ continuous.

Remark: 4.4 The converse of the above theorem need not be true as shown in the following example.

Example: 4.5 Let $U=\{a, b, c, d\}$ with $U / R=\{\{a\},,\{c\},\{b, d\}\} \quad$ and $\quad X=\{a, b\}$. Then $\tau_{R}(X)=\{U, \phi,\{a\},\{b, d\},\{a, b, d\}\}$.Let $\quad V=\{x, y, z, w\} \quad$ with $\quad V / R^{\prime}=\{\{x, y\},\{z\},\{w\}\} \quad$ and $Y=\{x, z\}$. Then $\sigma_{R^{\prime}}(Y)=\{V, \phi,\{z\},\{x, y\},\{x, y, z\}\}$. Define $f: U \rightarrow V \quad$ and $f \quad$ as $f(a)=z, f(b)=y, f(c)=x, f(d)=w . \quad$ Then $\quad f^{-1}(\{z\})=\{a\}, f^{-1}(\{x, y\})=\{b, c\}$, $f^{-1}(\{x, y, z\})=\{a, b, c\}$. Thus $\{a\},\{b, c\}$ and $\{a, b, c\}$ are $N g \beta$ open sets in $U$. That is the inverse image of every Nano open set in $V$ is $N g \beta$ open set in $U$. Therefore $f$ is $N g \beta$ continuous. But $f$ is not Nano g $\beta$ irresolute, since $f^{-1}(\{y, z, w\})=\{a, b, d\}$ which is not Nano g $\beta$ closed in $U$ whereas $\{y, z, w\}$ is Nano $g$ closed in $V$. Thus a Nano $g$ continuous function is not Nano $g$ irresolute.

Theorem: 4.6 A function $f: U \rightarrow V$ is Nano g $\beta$ irresolute if and only if for every Nano g $\beta$ open set $A$ in $V, f^{-1}(A)$ is Nano $g$ open in $U$.

Proof follows from the fact that the complement of a Nano g $\beta$ open set is Nano g $\beta$ closed and vice versa.

Theorem: 4.7 If $f: U \rightarrow V$ and $g: V \rightarrow W$ are both $N g \beta$ irresolute, then $g \circ f: U \rightarrow W$ is $N g \beta$ irresolute.

Proof: Let $A$ be $N g \beta$ open in $W$. Then $g^{-1}(A)$ is $N g \beta$ open in $V$, since $g$ is $N g \beta$ irresolute and $f^{-1}\left(g^{-1}(A)\right)=(g \circ f)^{-1}(A)$ is $N g \beta$ open in $U$, since $f$ is $N g \beta$ irresolute. Hence $g \circ f$ is $N g \beta$ irresolute.

Theorem: 4.8 If $f: U \rightarrow V$ is $N g \beta$ irresolute and $g: V \rightarrow W$ is $N g \beta$ continuous, then $g \circ f: U \rightarrow W$ is $N g \beta$ continuous.

Theorem: 4.9 If $f: U \rightarrow V$ is $N g \beta$ continuous nd $g: V \rightarrow W$ is Nano continuous, then $g \circ f: U \rightarrow W$ is $N g \beta$ continuous.

Proof of the theorem 4.8 and 4.9 are obvious.

Theorem: 4.8 If $f: U \rightarrow V$ is $N g \beta$ irresolute and $g: V \rightarrow W$ is Nano $\beta$ continuous, then $g \circ f: U \rightarrow W$ is $N g \beta$ continuous.

Proof: Let $A$ be Nano open in $W$. Then $g^{-1}(A)$ is Nano $\beta$ open in $V$, since $g$ is Nano $\beta$ continuous. Thus $g^{-1}(A)$ is $N g \beta$ open in $V$, since every Nano $\beta$ open set is $N g \beta$ open and $f^{-1}\left(g^{-1}(A)\right)=(g \circ f)^{-1}(A)$ is $N g \beta$ open in $U$, since $f$ is $N g \beta$ irresolute. Hence $g \circ f$ is $N g \beta$ continuous.

Similarly we can prove the following theorems. 
Theorem: 4.9 If $f: U \rightarrow V$ is $N g \beta$ irresolute and $g: V \rightarrow W$ is Nano semi continuous, then $g \circ f: U \rightarrow W$ is $N g \beta$ continuous.

Theorem: 4.10 If $f: U \rightarrow V$ is $N g \beta$ irresolute and $g: V \rightarrow W$ is Nano pre continuous, then $g \circ f: U \rightarrow W$ is $N g \beta$ continuous.

Theorem: 4.11 If $f: U \rightarrow V$ is $N g \beta$ irresolute and $g: V \rightarrow W$ is Nano regular continuous, then $g \circ f: U \rightarrow W$ is $N g \beta$ continuous.

Theorem: 4.12 If $f: U \rightarrow V$ is $N g \beta$ irresolute and $g: V \rightarrow W$ is Nano $\alpha$ continuous, then $g \circ f: U \rightarrow W$ is $N g \beta$ continuous.

Theorem: 4.13 If $f: U \rightarrow V$ is $N g \beta$ irresolute and $g: V \rightarrow W$ is Nano b continuous, then $g \circ f: U \rightarrow W$ is $N g \beta$ continuous.

Theorem: 4.14 If $f: U \rightarrow V$ is $N g \beta$ irresolute and $g: V \rightarrow W$ is Nano g continuous, then $g \circ f: U \rightarrow W$ is $N g \beta$ continuous.

Theorem: 4.15 If $f: U \rightarrow V$ is $N g \beta$ irresolute and $g: V \rightarrow W$ is Nano gs continuous, then $g \circ f: U \rightarrow W$ is $N g \beta$ continuous.

Theorem: 4.16 If $f: U \rightarrow V$ is $N g \beta$ irresolute and $g: V \rightarrow W$ is Nano gr continuous, then $g \circ f: U \rightarrow W$ is $N g \beta$ continuous.

Theorem: 4.17 If $f: U \rightarrow V$ is $N g \beta$ irresolute and $g: V \rightarrow W$ is Nano $\alpha$ g continuous, then $g \circ f: U \rightarrow W$ is $N g \beta$ continuous.

\section{References}

[1]. $\quad$ Abd EL-Monsef M. E., EL-Deep S. N. and Mahmoud R. A., $\beta$-Open Sets and $\beta$-continuous mappings, Bull. Fac. Sci., Assiut Univ., 12 (1983), 77-90.

[2]. Balachandran. K., Sundaram. P., and Maki. H., On generalized continuous maps in topological Spaces, Mem. Fac. Sci. Kochi Univ. Ser. A. Math., 12(5)(1991).

[3]. Bhuvaneswari. K., and Mythili Gnanapriya. K., Nano generalized closed sets in nano topological spaces,International Journal of Scientific and ResearchPublications,4(5)(2014)1-3.

[4]. Gnanmbal. Y., On Nano $\beta$ open sets, Int. Jr. of Engineering, 1(2) (2015), 1-6.

[5]. Lellis Thivagar .M., and Carmel Richard, On Nano forms of weakly open sets, International Journal of Mathematics and Statistics Invention, 1(1) (2013), $31-37$.

[6]. Lellis Thivagar .M., and Carmel Richard, On Nano Continuity, Mathematical Theory and Modeling, 3(7)(2013), 32-37.

[7]. Shalini. S.B., and Indirani. K., Characterisation of nano generalized $\beta$ closed sets in nano topological spaces, International Journal Sciences and Applied Research, 4(1)(2017), 7-11. 